Krzysztof Kotuła

Uniwersytet Marii Curie-Skłodowskiej w Lublinie

\title{
CREER UN ENVIRONNEMENT INTERACTIF EN CLASSE DE FLE
}

\begin{abstract}
Creating an interactive language classroom
Traditional communication in foreign language classes, even if varied by the use of diverse games and simulations, takes place in a sterile classroom environment, and therefore, is rarely encouraging. However, the new generation of pupils is growing up on computer games, and this fact is changing their expectations and demands on education. The dynamically changing virtual world of videogames may be the perfect environment for them to develop various skills. In this paper, I would like to present my preliminary conclusions made while implementing videogames into foreign language classes in a high school.
\end{abstract}

\section{Introduction}

II n'est pas exagéré de constater que les nouvelles technologies ont définitivement et irréversiblement transformé le modèle de travail de l'enseignant de langue étrangère. Naturellement, les élèves ont aussi été affectés par ce changement. De plus en plus nombreux sont les outils qui rendent leur travail plus efficace, leur permettent de collaborer entre eux, de réaliser activement des projets divers, ainsi que de développer leur compétences générales et linguistiques. Du point de vue du professeur, les nouvelles technologies permettent entre autres de gérer plus efficacement le travail de classe, de promouvoir l'autonomie de ses élèves, d'augmenter leur motivation et de s'assurer que l'enseignement a également lieu en milieu extrascolaire. Dans le présent article, nous allons placer au cœur de notre réflexion un élément qui est pratiquement indissociable des nouvelles technologies, à savoir l'interactivité. 
Nous allons présenter des outils qui permettent de transformer une classe de FLE en un environnement actif réagissant aux actions des apprenants.

\section{Le sage sur l'estrade et le guide-accompagnateur}

Prenons comme point de départ de notre réflexion la représentation schématique d'une classe de langue "classique", qui continue à être un environnement privilégié par de nombreux enseignants (Figure 1). Sans doute, cela pourrait être une illustration classique d'un modèle appelé fréquemment " sage sur l'estrade » (sage on the stage ; cf. King, 1993). Selon ce modèle, c'est le professeur qui occupe la place centrale dans le processus de l'enseignement et c'est lui qui détient le contrôle quasi-absolu de la manière dont la langue étrangère se manifeste en classe. Cela se produit aussi bien à travers ses propres élocutions (symbolisées par un phylactère dans notre schéma) que par l'intermédiaire de différents médias. C'est l'enseignant qui a une influence décisive sur la manière dont ces documents seront présentés, analysés et étudiés. Par exemple, il peut demander à ses étudiants de lire le texte de telle ou telle façon, d'observer les structures grammaticales qui s'y manifestent ou de s'en servir en tant qu'exercice de prononciation (lecture à voix haute). Quant aux enregistrements sonores, c'est le professeur qui va décider du nombre d'écoutes, choisir les passages les plus importants, etc. Il en est de même avec les films vidéo, qu'il peut présenter dans leur ensemble ou fragmentairement, avec ou sans son, ou bien en accéléré (cf. Kozieł, 2010). Même si l'enseignant doit en principe adapter son comportement aux réactions de la classe, cela ne change en rien le fait que les étudiants ne peuvent pas disposer librement des ressources en langue étrangère. Tout passe par l'intermédiaire du professeur, qui est le seul élément interactif proprement dit dans la classe.

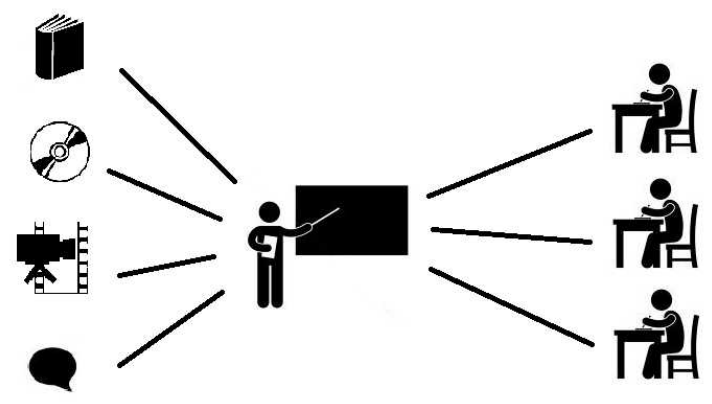

Figure 1 
Cette philosophie, on le sait, est depuis plusieurs années critiquée par de nombreux pédagogues qui lui préfèrent un modèle " guide-accompagnateur " (guide on the side; cf. King, 1993). Dans celui-ci, le professeur, souvent occupant une place parmi les élèves, les assiste dans leur travail, en devenant avant tout un facilitateur. Pourtant, comme l'a remarqué avec justesse Wiliam, " Le danger de cette caractérisation est qu'elle est souvent interprétée comme une exonération des responsabilités de l'enseignant quant aux acquisitions effectives des élèves. [...] Le rôle de l'enseignant est "d'organiser » un environnement d'apprentissage, tant du point de vue de sa conception que de son exploitation " (Wiliam, 2010 : 161). C'est justement le problème de l'organisation de l'environnement d'apprentissage qui va nous intéresser dans le présent article, et tout particulièrement la question de l'introduction des éléments interactifs dans la classe de langue.

Si nous sommes d'accord sur les avantages d'une configuration dans laquelle le professeur est un témoin et guide qui intervient principalement lorsque les apprenants ont besoin de son soutien, il faut s'interroger sur le modèle d'interaction enseignant - médias - élèves qui assurera un apprentissage efficace. Certes, beaucoup dépend de la volonté de l'enseignant. Son attitude, son ouverture d'esprit et sa détermination ainsi que ses compétences sont souvent la clé du succès. II faut cependant se rendre compte que c'est la nature même des médias utilisés traditionnellement en classe de langue qui impose souvent un certain modèle de travail. Qu'il s'agisse des enregistrements sonores ou des films vidéo, leur fonctionnement doit être forcément assuré par quelqu'un se trouvant dans la classe et il n'est pas étrange que dans la plupart des cas ce soit l'enseignant qui assume ce rôle. Pourtant, le développement des nouvelles technologies permet l'implantation dans la classe de FLE d'outils d'un nouveau type, se caractérisant par leur interactivité. Ceci est une vraie révolution, car l'échange d'informations peut se produire sans l'intermédiaire du professeur ; l'environnement interactif réagit et s'adapte aux actions des élèves d'une manière entièrement autonome. Cette situation permet au professeur de devenir un guide accompagnateur qui observe et facilite l'échange d'informations entre les apprenants sans être nécessairement l'initiateur de chaque action.

\section{L'environnement interactif en classe de FLE}

Quelle peut être la fonction d'un milieu interactif dans la classe de FLE ? En fait, ce type d'environnement est le seul qui recrée dans une certaine mesure des conditions de communication comparables sur le plan fonctionnel à celles 
de la vie réelle, dans laquelle chacune de nos élocutions entraîne une réaction ou transformation de notre entourage. Cela répond parfaitement aux besoins des apprenants qui, dans les conditions scolaires normales, sont totalement dépourvus de ce genre de stimuli. Loiseau décrit très bien ce problème: " Dans les classes de L.E., enseignants et apprenants pénètrent un monde linguistique et culturel différent de leur monde 'maternel', un monde virtuel car recréé artificiellement par des textes, des images ou des documents audiovisuels. Si certains apprenants de la L.E. ont parfois la chance de découvrir, dans sa réalité, le monde de la L.E., d'autres, nombreux, - et souvent, certains enseignants également - n'en connaîtront que la forme virtuelle » (Loiseau, 1997 : 64).

Loiseau touche ici au cœur du problème. Dans la classe traditionnelle, la communication entre les étudiants et le professeur se déroule dans un environnement stérile, dans lequel le monde de la L.E. n'est reproduit que par l'intermédiaire des éléments statiques et non interactifs. Ceci démotive forcément les apprenants qui souvent ne sont pas émotionnellement impliqués dans le processus d'acquisition de la langue étrangère. Comme nous l'avons dit plus haut, dans la vie réelle un succès communicatif nous apporte d'habitude un profit tangible. Dans la classe, le succès communicatif n'est récompensé que par un éloge du professeur ou une bonne note. Pour remédier en quelque sorte à cette situation, les activités de classe sont souvent conçues de sorte à simuler la vie réelle - il suffit de citer ici les exercices très populaires du type " jouez les scènes " dans lesquels les élèves sont priés d'incarner différents personnages et de dialoguer entre eux. II faut cependant admettre que ce genre d'exercices se déroule d'habitude d'une manière qui n'a rien à voir avec la communication réelle : questions et réponses stéréotypées, réactions prévisibles des interlocuteurs, absence de rétroaction active. Quant au monde virtuel de la L.E. mentionné par Loiseau, il n'est forcément pas interactif, car les documents sonores visuels et textuels ne sont qu'une reproduction figée des actes de communication.

II semble donc que les milieux interactifs soient une solution idéale dont l'application s'impose dans une classe moderne de FLE. Dans le présent article, nous allons nous concentrer sur un des outils que nous considérons comme le mieux approprié à favoriser un enseignement interactif de la L.E. : les jeux vidéo. Outre une réflexion théorique, nous allons présenter nos propres observations faites lors de l'implémentation des jeux dans les cours de la langue française au lycée. 


\section{Les jeux vidéo en classe de FLE}

La proposition d'utiliser les jeux vidéo en classe de langue sera sans doute considérée par plusieurs comme controversée. Même si la popularité de ce type de divertissement est incontestable parmi les jeunes - comme le montre le rapport Dzieci aktywne online (Les enfants actifs en ligne), 70\% des enfants polonais dans la tranche d'âge entre 4 et 14 ans utilisent un ordinateur, et ils le font avant tout pour jouer (cité d'après Błaszkiewicz, 2011 : 35) - les jeux sont traditionnellement considérés comme une activité qui n'est pas "sérieuse ". Pourtant, certains pédagogues prônent depuis longtemps les avantages de l'implémentation de la stratégie ludique dans le processus glottodidactique. Pour ne citer que quelques publications, SiekPiskozub (1995, 2001), par exemple, montre comment les jeux et les simulations peuvent compléter efficacement l'apprentissage d'une langue étrangère. Nombreuses sont aussi les voix, surtout outre-Atlantique, qui vont encore plus loin en clamant que les jeux vidéo sont beaucoup plus pédagogiquement utiles que l'école (Johnson, 2005, Prensky, 2006, Gee, 2007, Mawer et Stanley, 2007, Aldrich, 2009). II semble donc qu'en sousestimant l'utilité de ce type d'activités, nous commettons une grave erreur.

Il existe relativement peu de publications consacrées à l'emploi des jeux vidéo dans la classe de langue (pour une bibliographie à peu près complète cf. Li, Liu et Boyer, 2009 : 136, de Haan, 2011 : 46-47). En général, les spécialistes s'accordent à dire que leur impact sur le développement des compétences linguistiques est entièrement positif. Dans une de toutes récentes publications consacrées à ce sujet, Thomas constate que " les jeux peuvent être motivants, réduire le stress, permettre aux apprenants de se concentrer sur la communication dans la langue cible plutôt que sur l'emploi des structures linguistiques correctes, offrent aux étudiants introvertis davantage de possibilités de s'exprimer et créent une ambiance informelle qui augmente la réceptivité des élèves " ${ }^{1}$ (Thomas, $2012: 11$ ). Mawer et Stanley pour leur part soulignent que l'introduction des jeux vidéo en classe de langue permet de développer chez les élèves nombre de compétences et de comportements tels que "la capacité de résoudre créativement des problèmes, la prise de risques calculés, la persévérance, l'attention au détail

\footnotetext{
${ }^{1}$ Thomas, 2012 : 11 : games can be motivating, reduce anxiety, focus learners on communicating in the target language rather than on using correct linguistic structures, provide more introverted students with a greater range of opportunities for selfexpression and create an informal atmosphere that enhances learner receptiveness.
} 
ainsi que la collaboration efficace $»^{2}$ (Mawer et Stanley, $2011: 10$ ). Les avantages potentiels des jeux vidéo ne sont donc pas négligeables.

Quels sont les jeux que l'on peut adapter aux besoins d'une classe de FLE ? II paraît que les possibilités sont nombreuses. En général, les chercheurs travaillant dans ce domaine se concentrent sur les jeux qui contiennent de longues heures d'enregistrements sonores, tels que les jeux de rôle, dans lesquels le joueur incarne un personnage qu'il fait évoluer au fil d'une quête se déroulant dans un vaste univers (Cruz, 2007, Surdyk, 2008, de Haan, 2011), ou les jeux vidéo de simulation de vie, dans lesquels le joueur crée un personnage dont il doit gérer les besoins quotidiens en lui faisant mener la vie qu'il désire (Purushotma, 2005, Ranalli, 2007). Dans le cadre de notre réflexion sur l'interactivité, nous avons choisi une approche tout à fait différente. Nous allons nous concentrer sur des jeux d'un autre type, à savoir les jeux vidéo de réflexion.

Nous pourrions naturellement nous demander pourquoi adapter les jeux vidéo à la réalité de la classe de langue alors qu'existe un grand nombre de jeux conçus expressément dans le but de rendre l'enseignement du FLE plus efficace et divertissant (cf. p.ex. Krystalli, 2008). Le problème avec ce type de logiciels c'est qu'ils sont le plus souvent créés par des spécialistes en didactique des langues qui ignorent pour la plupart les facteurs qui rendent les jeux vidéo aussi attrayants. C'est précisément la raison pour laquelle les jeux créés pour faciliter l'enseignement d'une L.E. sont pour la plupart des tests maladroitement déguisés. Prendre comme point de départ un objectif pédagogique et construire un jeu autour de lui est toujours condamné à l'échec.

Si nous sommes d'avis que les jeux vidéo, en particulier ceux de réflexion, peuvent très bien convenir aux besoins d'une classe moderne de L.E., nous devons nécessairement répondre à la question : quelles sont leurs caractéristiques et en quoi sont-ils différents (mieux adaptés à la réalité d'une classe de langue) des autres jeux ? Tout d'abord, les jeux vidéo de réflexion se caractérisent par le fait qu'ils ne mettent pas l'accent sur la compétition entre I'homme et la machine ou bien entre les joueurs, mais plutôt sur la recherche d'une solution à un problème. Le défi peut prendre différentes formes : résolution d'énigmes, navigation, manipulation d'objets, etc. L'accent n'est pas mis sur les réactions rapides des joueurs mais plutôt sur leurs capacités intellectuelles, leur curiosité et leur créativité.

Contrairement aux autres types de jeux, les jeux de réflexion ne contiennent pratiquement pas d'instructions verbales. Ils présentent

\footnotetext{
${ }^{2}$ Mawer and Stanley, 2011 : 10 : creative problem solving, calculated risk taking, persistence, attention to detail [and] effective collaboration.
} 
également un tout autre type de défi que les jeux de rôle ou de simulation de vie. Le joueur n'est pas censé créer un personnage auquel il s'identifie et qui évolue dans le cadre d'une " histoire ". Dans la plupart des cas, tout ce que voit le joueur est un groupe d'objets virtuels qu'il doit manipuler d'une certaine façon pour atteindre le but prévu par les programmeurs. En raison de leur caractère, les jeux de réflexion favorisent d'une manière naturelle le travail collectif. La langue étrangère se manifeste ici d'une façon tout à fait particulière : ce n'est plus l'ordinateur qui communique avec le joueur mais les joueurs qui communiquent entre eux pour essayer de résoudre ensemble le problème auquel ils sont confrontés.

Le modèle de communication dans la classe se transforme donc entièrement: nous n'avons plus affaire à une configuration dans laquelle les joueurs, assis devant leurs ordinateurs, essaient de faire face individuellement aux défis du monde virtuel. Au contraire, ils créent une communauté dans laquelle ils échangent librement des idées. Cela permet de s'assurer que les élèves perfectionnent non seulement leurs compétences réceptives (ce qui a lieu dans le cas où les joueurs écoutent ce que disent les personnages virtuels et réagissent seulement aux commandes de l'ordinateur), mais aussi productives, car ils doivent décrire ce qu'ils voient, spéculer quant à la nature du monde virtuel et négocier chacun des mouvements.

Les autres avantages des jeux vidéo de réflexion ne sont pas non plus négligeables. Plusieurs d'entre eux sont accessibles gratuitement sur internet. Ils sont d'habitude réalisés en technologie flash et ne requièrent pas d'ordinateurs puissants pour fonctionner. Le défi présenté varie d'un jeu à l'autre, ce qui permet aux élèves de stimuler le développement des compétences communicatives de façon différente. Les règles de ces jeux sont d'habitude simples, ce qui est important, car un jeu difficile pourrait facilement décourager les élèves. Finalement, il n'y a pas de limite de temps, ce qui est particulièrement important dans le contexte où chacun des joueurs devrait avoir le temps de se prononcer sur la manière la plus efficace de résoudre un problème. Nous présentons le déroulement d'une partie hypothétique dans un de nos articles (Kotuła, 2012).

L'approche collective nécessite forcément la création d'une aire de jeu commune. Le tableau interactif s'avère le meilleur outil dans ce contexte. Cet outil, présent dans un nombre croissant d'écoles, peut d'une part servir de grand écran sur lequel les joueurs peuvent suivre confortablement le déroulement de la partie, d'autre part, grâce à ses propriétés interactives, il permet d'effacer - même si ce n'est que partiellement - la frontière entre le monde réel et virtuel. En effet, l'étudiant a la possibilité de manipuler les objets du jeu avec le toucher. Un 
étudiant peut dire à un autre de déplacer, tirer, pousser ou tourner un objet et son partenaire va tout simplement le faire. Dans les jeux, les objets sont présentés souvent comme s'ils étaient réels, leur manipulation est accompagnée d'effets visuels et sonores et leurs mouvements sont régis par les lois de la physique, tout cela fait que le joueur est émotionnellement impliqué dans l'ensemble des activités.

C'est justement là que se manifeste l'énorme avantage des jeux numériques face aux jeux non-numériques. Comme les règles du jeu sont inhérentes au monde virtuel et se manifestent spontanément au cours de la partie, le professeur n'est plus obligé d'expliquer à ses élèves ce qu'ils doivent faire pour remporter un succès. Ainsi, les apprenants doivent découvrir euxmêmes les règles du jeu. De cette manière, ils n'ont plus le sentiment de participer à un évènement organisé et contrôlé par l'enseignant qui décide quel mouvement est permis ou non, s'assure que les règles sont obéies, supervise le déroulement de la compétition et déclare arbitrairement telle ou telle équipe victorieuse. Dans le modèle en question (Figure 2), il supervise uniquement le flux d'informations dans la classe et, si nécessaire, joue le rôle de facilitateur dans les moments critiques. Ceci est très motivant car les étudiants ont l'impression de participer à un événement authentique.

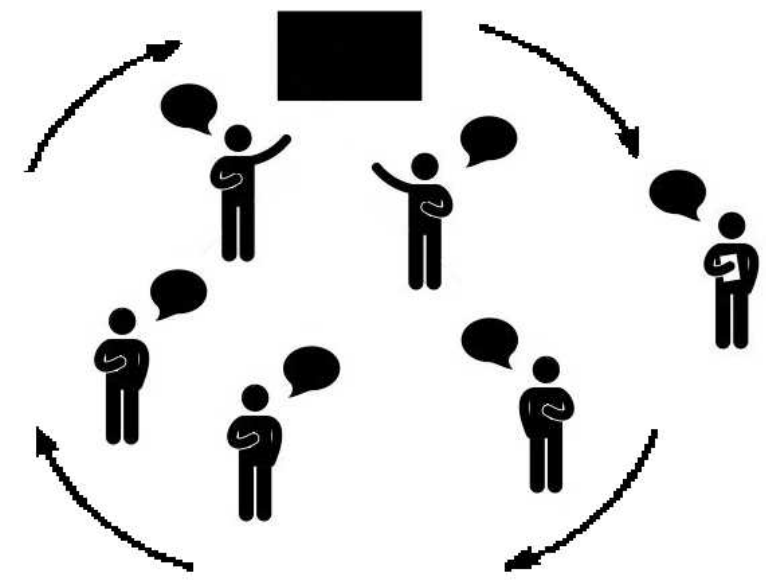

Figure 2

\section{Conclusion}

Il faut bien sûr se rendre compte que l'implémentation des jeux vidéo dans le processus glottodidactique n'est pas une tâche facile. Comme le remarque avec justesse de Haan, «le potentiel des jeux vidéo dans l'apprentissage ne réside 
pas nécessairement en eux-mêmes (c'est-à-dire qu'un étudiant essaie d'apprendre une L.E. en jouant à un jeu) mais plutôt dans le contexte et les activités relatives et découlant du jeu $\|^{3}$ (de Haan, 2011 : 46). II serait déraisonnable de croire qu'en laissant simplement les élèves jouer aux jeux vidéo, nous allons automatiquement faciliter le développement de leurs compétences linguistiques. La présence de l'enseignant est un élément absolument nécessaire de l'équation. Ce qui pose réellement problème, c'est la manière dont sa présence se manifeste dans la classe. II doit superviser le flux d'informations tout en se rendant compte que l'excès de contrôle étouffera à coup sûr l'enthousiasme des étudiants. Un équilibre doit donc être établi entre le temps du jeu et celui qui sera consacré aux autres activités, et les deux doivent se compléter harmonieusement. C'est la raison pour laquelle de nombreux enseignants - ceux pour qui le monde des jeux vidéo et des innovations techniques est un terrain inconnu, tout comme ceux qui se sentent mal à l'aise dans la situation où il faut quitter le cadre rigide imposé par les activités de classe traditionnelles - seront probablement réticents à l'idée d'introduire ce type d'innovations. II faut cependant se rendre compte que personne n'arrêtera le progrès technologique et que les nouvelles technologies, qu'on le veuille ou non, vont irréversiblement transformer la classe de langue.

\section{BIBLIOGRAPHIE}

Aldrich, C. 2009. Learning Online with Games, Simulations and Virtual Worlds. San Francisco: Jossey-Bass.

Błaszkiewicz, R. 2011. „Gry komputerowe a zdrowie dziecka w młodszym wieku szkolnym". Nauczanie Poczqtkowe XXXIV, 1: 34-43.

Cruz, J. Q. 2007. „Video Games and the ESL Classroom”. The Internet TESL Journal Vol. XIII, No. 3, http://iteslj.org/Articles/Quijano-VideoGames.html DW 13.09.2012. deHaan, J. 2011. „Teaching and learning English through digital game projects”. Digital culture \& education III, 1: 46-55.

Dylan, W. 2010. „Le rôle de l'évaluation formative dans les environnements d'apprentissage efficaces" (in) Comment apprend-on ? La recherche au service de la pratique (éd. H. Dumont, D. Istance et F. Benavides). Éditions OCDE: 143-169.

Gee, J. P. 2007. What video games have to teach us about learning and literacy. New York: Palgrave Macmillan.

\footnotetext{
${ }^{3}$ De Haan, $2011: 46$ : the power of games for educational purposes may not reside in the games themselves (i.e., a language student trying to learn the second language from a digital game), but rather in the context and activities related to and extending from play.
} 
Johnson, S. 2005. Everything bad is good for you: how today's popular culture is actually making us smarter. New York: Riverhead Books.

King, A. 1993. „From Sage on the Stage to Guide on the Side”. College Teaching XXXXI, 1: 30-35.

Kotuła, K. 2012. „Gra komputerowa na lekcji języka obcego”. Języki Obce w Szkole 3: 122-124.

Kozieł, R. 2010. „Ewaluacja dydaktyzacji filmów w wybranych podręcznikach do nauki języka niemieckiego" (in) Materiały edukacyjne w nauczaniu języków obcych - teoria i praktyka (red. K. Droździał-Szelest). Gorzów Wielkopolski: Wyd. Państwowej Szkoły Zawodowej w Gorzowie Wielkopolskim: 71-78.

Krystalli, P. 2008. „Les jeux vidéo en classe de FLE” (in) Année européenne du dialogue interculturel: communiquer avec les langues-cultures. Actes du Congrès international. Université Aristote de Thessalonique: 336-348.

Li, Z., Liu, F. et Boyer, J. 2009. „Amusing Minds for Joyful Learning through E-Gaming” (in) Handbook of research on e-learning methodologies for language acquisition (éd. R. Marriott et P. Torres). Hershey: Information Science Reference: 132-150.

Loiseau, Y. 1997. „De l'incidence de l'espace et du temps dans l'enseignement/apprentissage des langues étrangères" (in) Le FLE, un métier, une passion: réflexions en didactologie des langues-cultures (éd. M.-A. Nachez et S. Roch-Veiras). Angers: Université Catholique de l'Ouest: 59-86.

Mawer, K. et Stanley, G. 2011. Digital play: computer games and language aims. Surrey: Delta Publishing.

Prensky, M. 2006. Don't bother me Mom, I'm learning! How computer and video games are preparing your kids for twenty-first century success and how you can help! St. Paul: Paragon House.

Purushotma, R. 2005. „Commentary: You're not studying, you're just...”. Language Learning \& Technology IX, 1: 80-96.

Ranalli, J. 2008. „Learning English with The Sims: exploiting authentic computer simulation games for L2 learning". Computer Assisted Language Learning XXI, 5: 441-455.

Siek-Piskozub, T. 1995. Gry, zabawy i symulacje w procesie glottodydaktycznym. Poznań: Uniwersytet im. Adama Mickiewicza.

Siek-Piskozub, T. 2001. Strategia ludyczna w nauczaniu języków obcych, Warszawa: PWN.

Surdyk, A. 2008. „Klasyfikacja interakcji w grach typu role-playing games oraz relacje komunikacyjne i dydaktyczne w technice gier fabularnych". Homo Communicativus IV , 2: 115-125.

Thomas, M. 2012. „Contextualizing Digital Game-Based Language Learning: Transformational Paradigm Shift or Business as Usual?" (in) Digital Games in Language Learning and Teaching (éd. H. Reinders). New York: Palgrave Macmillan: 11-31. 